\title{
Interferometric out-of-focus imaging simulator for irregular rough particles
}

\author{
M. Brunel \\ marc.brunel@coria.fr \\ S. Coetmellec \\ G. Gréhan \\ H. Shen
}

\begin{abstract}
Departement d'Optique et Laser, UMR CNRS 6614 CORIA, Av. de l'Université, 76801 Saint-Etienne du Rouvray cedex, France

Departement d'Optique et Laser, UMR CNRS 6614 CORIA, Av. de l'Université, 76801 Saint-Etienne du Rouvray cedex, France

Departement d'Optique et Laser, UMR CNRS 6614 CORIA, Av. de l'Université, 76801 Saint-Etienne du Rouvray cedex, France

Departement d'Optique et Laser, UMR CNRS 6614 CORIA, Av. de l'Université, 76801 Saint-Etienne du Rouvray cedex, France
\end{abstract}

We present the development of an original simulator to predict interferometric out-of-focus patterns created by irregular rough particles. Despite important simplifications of the scattering properties, this simulator allows to predict quantitative properties of the speckle-like patterns: i.e. the dimension of the central peak of the $2 \mathrm{D}$-autocorrelation of the pattern. This parameter can then be linked to the size and the shape of the particle projected on the CCD sensor, in cases where there is no exact theoretical formulation to calculate the scattered intensity. An experimental demonstration is performed with irregular $\mathrm{NaCl}$ salt crystals.

[DOI: http://dx.doi.org/10.2971/jeos.2014.14008]

Keywords: Interferometric out-of-focus imaging, speckle, irregular particles, ABCD transfer matrices

\section{INTRODUCTION}

Interferometric out-of-focus imaging is a robust technique that has important potentiality for the characterization of droplets or bubbles [1]-[6]. Based on the scattering properties of particles $[7,8]$, it envolves simply a laser sheet and an imaging system (objective and CCD sensor). It can give access simultaneously to the position and the size of the objects under study [9], can be coupled to other techniques as PIV for velocity measurements [10]. The interpretation of the results is based on simple analytical relations [11]. They can be extended to more complex imaging systems through a description in terms of optical ABCD transfer matrices [12]. It is possible to perform optical compressing of the images to enhance the range of concentrations measurable [13], to develop realtime image processing algorithms [14], or to design specific imaging configurations for the analysis of droplets or bubbles in a 3D volume [15]-[17]. Unfortunately, the potentiality of this technique to study irregular scattering objects is not established and much has to be done in this direction. The main difficulty is that we need first to be able to describe the scattering properties of irregular objects. Unfortunately, this problem has no analytical solution in the framework of the Lorenz-Mie theory and can not be resolved in most of the cases. In the present study, we present the development of a simulator to predict some physical properties of the interferometric out-offocus patterns produced by irregular objects. Although we do not describe the scattering properties of the objects exactly, we can predict some statistical properties of the out-of-focus images and deduce some morphological properties of the objects under study (size, length, width). In a first section we present the simulator that we have developed, and the assumptions it is based on. As the rigorous description of the scattering properties of many complex objects has no exact solution, our method consists of describing complex irregular objects by a random location of glare points along the global form of these objects. Although our description of the scattering properties is not exact, the images that we predict contain exact informations about the particle under study. In the following section, we show indeed that we can evaluate the dimension of the irregular particle through the analysis of the second-order 2D-autocorrelation of speckle-like patterns. Analytical expressions are established that link the characteristics of specklelike patterns to the dimension of the irregular object. In a last section, these predictions are compared to experimental outof-focus images recorded with irregular $\mathrm{NaCl}$ salt crystals. Experiments show the validity of the procedure.

\section{ANALYTICAL THEORY OF INTERFEROMETRIC OUT-OF-FOCUS IMAGING}

\subsection{Description of the scattering objects}

Figure 1 shows a typical ILIDS experimental set-up (Interferometric Laser Imaging for Droplet Sizing). A pulsed laser sheet is sent towards a group of droplets. The forward scattered light is collected by a receiving optics. Different set-up at different off-axis angles $\Theta$ have been already investigated in the literature: at $45^{\circ}$ in references [4], $73^{\circ}$ in [6] or $90^{\circ}$ in 


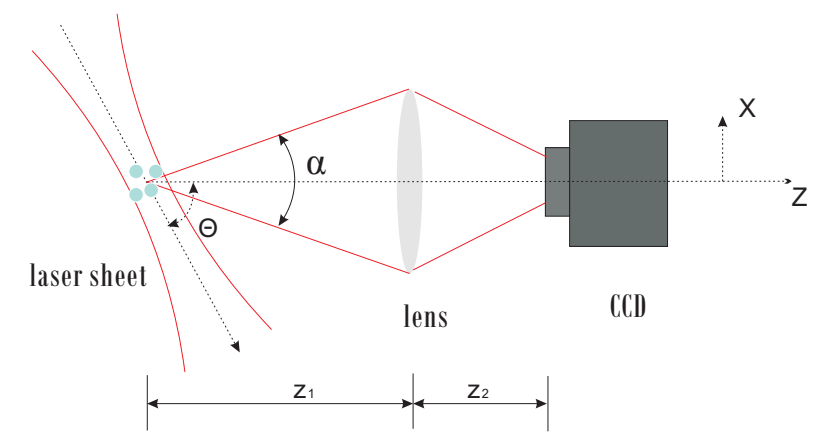

FIG. 1 Typical ILIDS configuration

[18]. Actually, ILIDS works in a large domain of forward scattering angles, from $30^{\circ}$ to $90^{\circ}$ [3]. The images are captured by a CCD camera positioned on a non-focal plane, where interference fringes appear (out-of-focus imaging). The intensity's oscillations (fringes) measured depend on the diameter of the droplets [11].

Let us now consider other objects than spherical droplets or bubbles. In our simulator, the irregular scattering objects will be described by an ensemble of $N_{g p}$ punctual glare points. The description of a spherical droplet or a spherical bubble is particularly convenient with this formalism. In this case, the number of glare points can be limited to 2, i.e. $N_{g p}=2$ $[19,12]$. Their position is given by simple analytical relations, using a geometrical optics approximation [11], and the experimental results are in very good concordance with simulations [12]. The description of irregular objects is more complex. The rigorous description of the scattering properties of irregular objects using exact Lorenz-Mie theory does not have exact solutions in a wide number of cases. However, we think that, although we are not able to describe rigorously the scattering properties, we are able to predict some statistical properties of the scattered images using a simplified approach. Our method consists of describing complex irregular objects by a random location of glare points along the global form of the irregular objects under consideration. Figure 2 shows some examples of irregular objects that we describe: a stick (a), an hexagon (b), an hexagonal plate (c), a cross (d), an ellipsoide (e), a sphere (f) (the 3D is not clearly visible on these planar figures but it is taken into account). In all cases, we realize a random positionning of $N_{g p}$ glare points along the pre-defined form. We have thus developed a MATLAB library of predefined objects (Figure 2 shows some typical examples). For each object, we can choose in our simulator:

- the form of irregular object and its dimension (see Figure 2), - the number of glare points to define the particle $\left(N_{g p}\right)$,

- the amplitude of the electric field emitted by each glare point $\left(\alpha_{j}\right)$,

- the phase of each glare point $\left(\varphi_{j}\right)$,

The 3D position of each glare point $\left(a_{j}, b_{j}, c_{j}\right)$ is defined randomly over the pre-defined form. In the simulations that will be presented in the next sections, the amplitude of all glare points will be identical. We are interested in the characterization of particles in the size domain from a tenth of micrometers to hundreds of micrometers. For spherical droplets, the phase shift introduced between the two glare points (which represent the reflected beam and the beam refracted by the
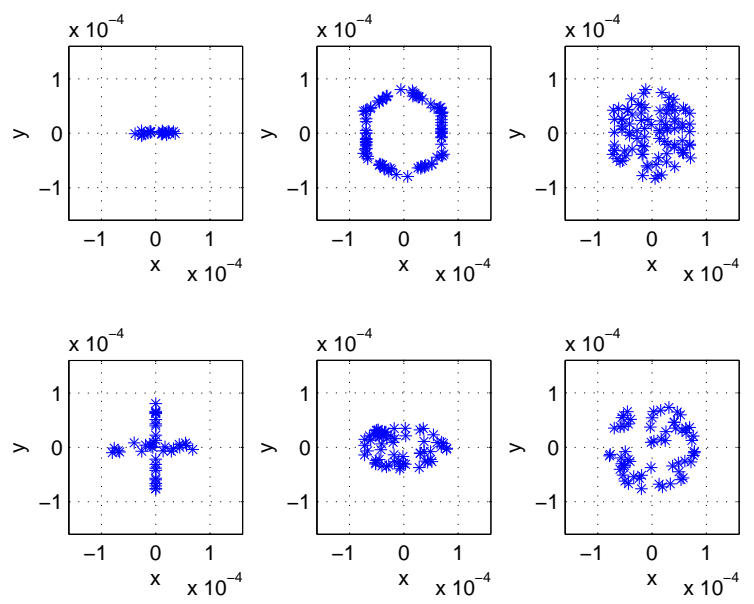

FIG. 2 Possible irregular objects

droplet without any internal reflection) is then much higher than $2 \pi$. In the case of irregular particles, we will assume that the phase of each glare point is a random variable in the range $[0,2 \pi]$. These two hypothesis could be easily modified. Once the irregular particle is defined, we can further choose:

- the orientation in space of the global irregular particle (using rotation matrices defined using MATLAB).

- the transverse and longitudinal (along the imaging z-axis) position in space of the particle.

These different parameters are then used to calculate the interferometric out-of-focus images as will be now presented.

\subsection{Analytical relations}

In the simplified scalar approach that we develop, the field emitted by the scattering particle is thus approached by $N_{g p}$ punctual light sources randomly located all over the particle such that the total electric field emitted by the global particle can be written:

$$
G_{0}(x, y, z)=\sum_{j=1}^{N_{g p}} \alpha_{j} \delta\left(x-a_{j}, y-b_{j}, z-c_{j}\right) e^{i \varphi_{j}}
$$

where the $\delta$ functions are Dirac functions. $N_{g p}$ is the number of emitters to be considered. $a_{j}, b_{j}$ and $c_{j}$ give the positons of the glare points, $\alpha_{j}$ is the amplitude of the electric field emitted by the glare point $j$, and $\varphi_{j}$ is the phase of the emitting glare point $j$. With this primary assumption, we can then calculate the expression of the electric field in the plane of the CCD sensor. The first step consists of calculating the expression of the electric field in the plane where the aperture-defining lens is located. According to the studies of [12], [20]-[22], it is calculated with the generalized Huygens-Fresnel integral. We obtain :

$$
G_{1}\left(\xi, \eta, \ell_{1}\right)=\sum_{j=1}^{N_{g p}} \frac{\alpha_{j} e^{i \frac{2 \pi}{\lambda}\left(n_{\ell_{1, j} \ell_{1, j}}\right)}}{i \lambda \sqrt{B_{1, j}^{x} B_{1, j}^{y}}} e^{i\left(\theta_{j}+\varphi_{j}\right)}
$$


where we note:

$$
\begin{aligned}
\theta_{j}=\frac{\pi}{\lambda}\left[\frac{1}{B_{1, j}^{x}}\right. & \left(A_{1, j}^{x} a_{j}^{2}-2 a_{j} \xi+D_{1, j}^{x} \xi^{2}\right) \\
& \left.+\frac{1}{B_{1, j}^{y}}\left(A_{1, j}^{y} b_{j}^{2}-2 b_{j} \eta+D_{1, j}^{y} \eta^{2}\right)\right]
\end{aligned}
$$

The different $A_{1, j^{\prime}}^{p}, B_{1, j}^{p}, C_{1, j^{\prime}}^{p} D_{1, j}^{p}$ (with $p=x$ or $p=y$ ) are the coefficients of the transfer matrices between the emitter $j$ of the scattering particle and the plane where the pupil is located. $\ell_{1, j}$ is the distance between the droplet and the plane of the pupil. $n_{\ell_{1, j}} \ell_{1, j}$ is the optical path between the emitter $j$ and the plane of the aperture. We can define two different transfer matrices for both transverse axes $x$ and $y$, which allows to describe cylindrical geometries.

We note $T(\xi, \eta)$ the transmission coefficient of the pupil. $T(\xi, \eta)$ equals 1 within a disk of radius $R_{0}$ (the radius of the aperture-defining lens), and equals 0 outside. $T(\xi, \eta)$ can be expressed as a superposition of Gaussian functions, as was proposed by [23], such as:

$$
T(\xi, \eta)=\sum_{k=1}^{N} P_{k} \exp \left[-\frac{Q_{k}}{R_{0}^{2}}\left(\xi^{2}+\eta^{2}\right)\right] .
$$

where the coefficients $P_{k}$ and $Q_{k}$ are given in references [23], and parameter $N$ equals 10 . The expression of the electric field in the plane of the aperturing pupil $G_{1}\left(\xi, \eta, \ell_{1}\right)$ is given by Eqs. (2) and (3). This Gaussian decomposition of $T(\xi, \eta)$ is very convenient because it allows to establish analytical expression of the diffracted pattern in the CCD plane. Let us now consider the propagation after the aperture. This part is described by complex matrices $M_{2}^{p}(p=x$ or $y)$. In this generalized case, we obtain :

$$
\begin{aligned}
& G_{2}\left(x^{\prime}, y^{\prime}, \ell\right)=\sum_{j=1}^{N_{g p}} \sum_{k=1}^{N}\left[\frac{e^{i \frac{2 \pi}{\lambda}\left(n_{\ell_{1, j}} \ell_{1, j}+n_{\ell_{2}} \ell_{2}\right)} e^{i \frac{\pi}{\lambda}\left(\frac{D_{2}^{x} x^{\prime 2}}{B_{2}^{x}}+\frac{D_{2}^{y} y^{\prime 2}}{B_{2}^{y}}\right)}}{(i \lambda)^{2} \sqrt{B_{1, j}^{x} B_{1, j}^{y} B_{2}^{x} B_{2}^{y}}}\right. \\
& \left.\times \frac{P_{k} \pi}{\sqrt{\gamma_{x, j}(k) \gamma_{y, j}(k)}} \alpha_{j} e^{\beta_{j}(k)} e^{i \varphi_{j}}\right]
\end{aligned}
$$

where:

$$
\begin{aligned}
\beta_{j}(k)=i \frac{\pi A_{1, j}^{x} a_{j}^{2}}{\lambda B_{1, j}^{x}}+i \frac{\pi A_{1, j}^{y} b_{j}^{2}}{\lambda B_{1, j}^{y}}-\frac{\phi_{x^{\prime}, j}^{2}}{4 \gamma_{x, j}(k)}-\frac{\phi_{y^{\prime}, j}^{2}}{4 \gamma_{y, j}(k)}, \\
\gamma_{x, j}(k)=\frac{Q_{k}}{R_{0}^{2}}-i \frac{\pi}{\lambda}\left(\frac{D_{1, j}^{x}}{B_{1, j}^{x}}+\frac{A_{2}^{x}}{B_{2}^{x}}\right), \\
\gamma_{y, j}(k)=\frac{Q_{k}}{R_{0}^{2}}-i \frac{\pi}{\lambda}\left(\frac{D_{1, j}^{y}}{B_{1, j}^{y}}+\frac{A_{2}^{y}}{B_{2}^{y}}\right), \\
\phi_{x^{\prime}, j}=\frac{2 \pi}{\lambda}\left(\frac{a_{j}}{B_{1, j}^{x}}+\frac{x^{\prime}}{B_{2}^{x}}\right), \\
\phi_{y^{\prime}, j}=\frac{2 \pi}{\lambda}\left(\frac{b_{j}}{B_{1, j}^{y}}+\frac{y^{\prime}}{B_{2}^{y}}\right) .
\end{aligned}
$$

$\left(n_{\ell_{2}} \ell_{2}\right)$ represents the optical path way between the aperture and the CCD sensor. The different $A_{2}^{p}, B_{2}^{p}, C_{2}^{p}, D_{2}^{p}$ (with $p=x$ or $p=y$ ) are the coefficients of the transfer matrices between the plane of the aperture and the plane of the CCD sensor.

\section{SIMULATION OF INTERFEROMETRIC OUT-OF-FOCUS IMAGES}

\subsection{Speckle-like patterns}

Using the Eqs. (5)-(10) we are then able to predict the interferometric out-of-focus image obtained for any pre-defined object of our MATLAB library. It is well known that the out-offocus image of a droplet is a circular spot filled with parallel dark and bright fringes. A droplet is indeed described by two glare points which can be assimilated to 2 coherent Dirac emitters as demonstrated in reference [12]. Note that all simulations that we could do in the past using this formulation with spherical droplets or bubbles, were prefectly corroborated by experiments in a wide range of configurations [12, 16, 17]. In the case of a droplet (or a bubble) represented by two emitting glare points, the frequency $F$ of the fringes is given versus the diameter of the droplets, and the characteristics of the imaging system through relation [12]:

$$
F=\left|\frac{a_{1}-a_{2}}{\lambda B_{\text {tot }}^{x}}\right|
$$

where $B_{\text {tot }}^{x}$ is the $B$-coefficient of the total transfer matrix (along axis $x$ ) between the plane of the scattering droplet $(z=0)$ and the plane where the CCD sensor is located $\left(z=z_{1}+z_{2}\right) \cdot\left(a_{1}-a_{2}\right)$ is the distance (along the $x$-axis) between the two glare points. Using a geometrical optics approximation, it is given by relation [11]:

$$
a_{1}-a_{2}=\frac{d}{2}\left(\cos (\Theta / 2)+\frac{m \sin (\Theta / 2)}{\sqrt{m^{2}+1-2 m \cos (\Theta / 2)}}\right)
$$

where $d$ is the diameter of the droplet, $m$ its index, and $\Theta$ is the scattering angle.

Figure 3 shows a second example: we consider an irregular stick. The stick is assimilated to 15 glare points randomly located in a parallelepipede whose dimensions are $80 \mu \mathrm{m} \times 10 \mu \mathrm{m} \times 10 \mu \mathrm{m}$. Numerically, the imaging set-up between the scattering element and the CCD sensor is as follows: free space propagation along distance $z_{1}=0.13 \mathrm{~m}$, propagation through a spherical thin lens (focus length $f=0.1 \mathrm{~m}$ ), free space propagation along distance $z_{2}=0.393 \mathrm{~m}$ (defocus parameter $\Delta p=-0.04 \mathrm{~m}$ ). The aperture-defining element is the lens, whose radius is $R_{0}=10 \mathrm{~mm}$. The wavelength is $532 \mathrm{~nm}$. This system is of revolution around the longitudinal $z$-axis. The different optical transfer matrices satisfy: $M_{1, j}^{x}=M_{1, j}^{y}$ (from $j=1$ to $j=N_{g p}$ ) and $M_{2}^{x}=M_{2}^{y}$. Note that our theoretical simulator could allow to describe cylindrical imaging systems. Figure 3(a) shows the disposition of the randomly located glare points while Figure $3(\mathrm{~b})$ shows the interferometric out-of-focus image theoretically predicted using Eqs. (5)-(10). The pattern looks like an asymmetric speckle pattern. For the analysis of speckle patterns, the second-order $2 \mathrm{D}$-autocorrelation is a very convenient image processing tool 
(a)
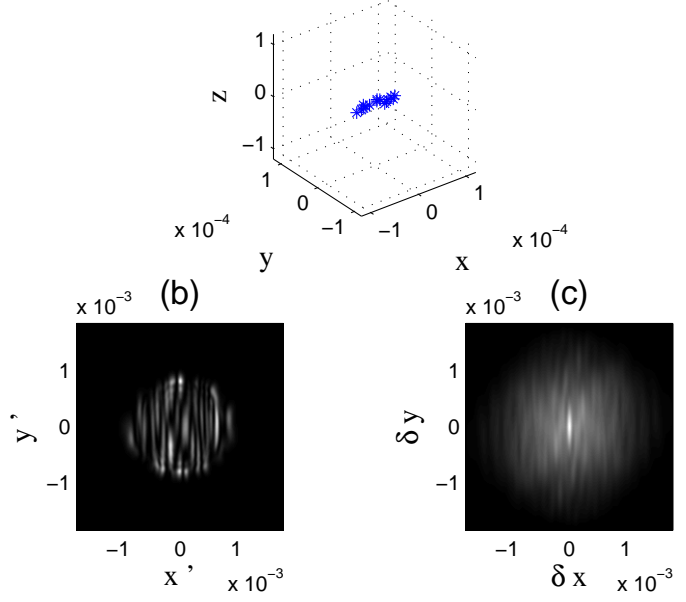

FIG. 3 Interferometric out-of-focus imaging of a stick represented by 15 emitters: location of the 15 emitters randomly located along a stick (a), corresponding out-of-focus image (b) and its 2D-autocorrelation (c) (all dimensions in meters).

[24]. It is defined by:

$$
A[I](\delta x, \delta y)=\iint_{-\infty}^{+\infty} I(x, y) \cdot I^{*}(x-\delta x, y-\delta y) d x d y
$$

In order to characterize this speckle pattern, we plot on Figure 3(c) the 2D-autocorrelation of the pattern of Figure 3(b). The central peak of the 2D-autocorrelation function gives an estimation of the dimension of the speck of light [24]. The asymmetry of the peak is due to the geometry of the scattering element: a parallelepipedic stick. To summarize, the longer the extension of the element (along the x-axis in this case), the smaller the dimension of the central peak of the autocorrelation trace, and reciprocally (which is the case along the y-axis). This effect will be quantified in the next sub-section.

In order to understand the influence of the number of glare points that are considered, we plot on Figure 4(a) and 4(b) the representation of this stick using 15 glare points or 30 glare points. The optical system is the same as previously (parameters $z_{1}=0.13 \mathrm{~m}, f=0.1 \mathrm{~m}, z_{2}=0.393 \mathrm{~m}, R_{0}=10 \mathrm{~mm}$ ). We plot then on Figure 4(c) and 4(d) the 2D-autocorrelations of the speckle patterns that are calculated in both cases. The speckle patterns are completely different in both cases and can not be compared (they are not reported here), but their 2Dautocorrelations can be compared. In particular the dimension of the central peak is only slightly modified. This can be seen more explicitely on Figure 5 where we plot the profile of the 2D-autocorrelation-peak along the x-axis (Figure 5(a)) and along the y-axis (Figure 5(b)). The curves are calculated for a parallelepipedic stick whose dimensions are in all cases : $80 \mu \mathrm{m} \times 10 \mu \mathrm{m} \times 10 \mu \mathrm{m}$, but considering 15 (solid lines), 30 (dashed lines) or 50 (dotted lines) randomly-located glare points in the stick. Note that they correspond to Figure 4, except the case with 50 glare points which had not been added to Figure 4 for sake of clarity. $\epsilon$ is the length of the stick: $\epsilon=80 \mu \mathrm{m}$. The background of the curves (pedestal) is different in all cases, but the differences in the width of the central peak do not exceed $10 \%$ between the 3 values $N_{g p}$ that are considered. This is verified both along the horizontal $\mathrm{x}$-axis (a)

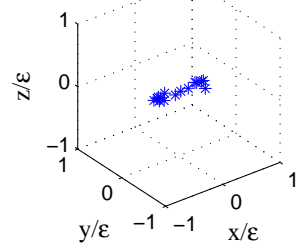

(c)

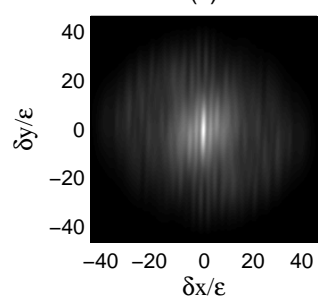

(b)

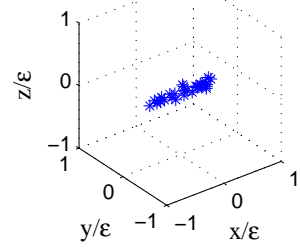

(d)

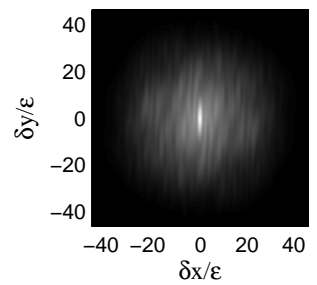

FIC. 4 Influence of the number of emitters: location of 15 emitters (a) and the corresponding $2 \mathrm{D}$-autocorrelation of the out-of-focus image predicted (c), location of 30 emitters (b) and the corresponding $2 \mathrm{D}$-autocorrelation of the out-of-focus image predicted (d). $\epsilon$ is the length of the stick: $\epsilon=80 \mu \mathrm{m}$ (a)

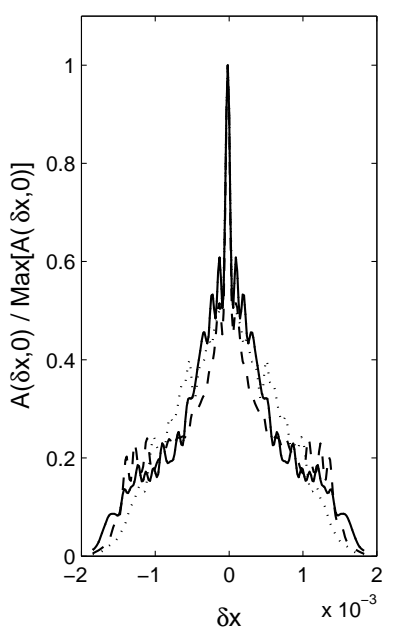

(D)

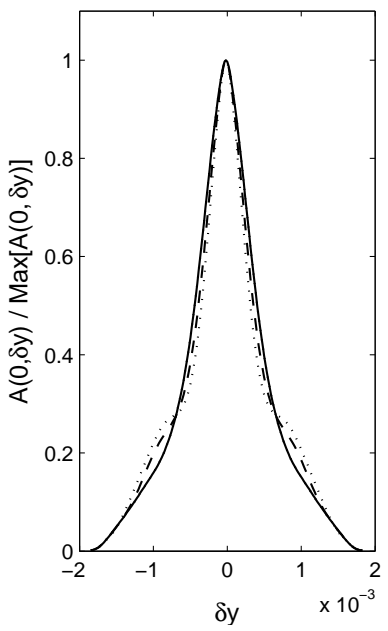

FIC. 5 x-cut (a) and y-cut (b) of the central peak of the 2D-autocorrelation images considering 15 emitters (solid lines), 30 emitters (dashed lines) or 50 emitters (dotted lines).

(Figure 5(a)) and along the vertical y-axis (Figure 5(b)). Let us now quantify these first observations.

\subsection{Quantitative analysis of the speckle-like patterns}

The aim of our study is to give the way to determine the dimension of the irregular particle under observation (and eventually some information concerning its morphology) from the observation of the interferometric out-of-focus images. As could be previously mentioned, a powerful parameter to be considered is the size of the speck of light in the speckle patterns. As will be shown now, it is possible to quantify the speck's size of the speckle pattern versus the dimension of the irregular particle and the parameters of the out-of-focus imaging set-up, with a relatively good precision. In the case of particles represented by glare points located randomly (but uniformly) along the particle, we note $\Delta$ the distance between the 
two farthest glare points (along axis $\mathrm{x}$ ). We will generalize relation (11). Neglecting the diffractin rings due to the aperture, the highest frequency present in the speckle pattern along the axis $x$ is given by

$$
F=\left|\frac{\Delta}{\lambda B_{\text {tot }}^{x}}\right|
$$

For the imaging system that we have already considered (i.e. with $\left.B_{\text {tot }}^{x}=0.0121\right)$, Figure 6 shows $1 / F$ versus $\Delta$ in black solid line. $1 / F$ has the dimension of meters and will now be shown to be related to the speck's size. Let us consider the characteristics of speckle-like out-of-focus images calculated using relations (5)-(10). The central peak of the 2D-autocorrelation of speckle patterns gives a statistical estimation of the size of the speck of light. Using relations (5)-(10), we have calculated approximately 70 speckle-like out-of-focus patterns for 70 different stick-like particles. Each pattern is associated to a particle represented by 15 glare points randomly located in a parallepipedic stick of dimensions Length $\mu \mathrm{m} \times 10 \mu \mathrm{m} \times 10 \mu \mathrm{m}$ (Length along the $\mathrm{x}$-axis, $10 \mu \mathrm{m}$ along the $\mathrm{y}$ and $\mathrm{z}$ axes). We change parameter Length in the range $15 \mu \mathrm{m}-200 \mu \mathrm{m}$. From these 70 out-of-focus patterns, we have calculated their $702 \mathrm{D}$ autocorrelation images, and measured the width of the central peak of the $702 \mathrm{D}$-autocorrelations along the $\mathrm{x}$-axis (the width is evaluated between the two points at $70 \%$ of the peak's maximum in order to be sure that we do not evaluate the width of a pedestal). For each of these patterns, we have reported on Figure 6 (crosses) the width of the 2D-autocorrelation peak along the x-axis versus the farthest distance along $\mathrm{x}$, noted $\Delta$, between two glare points. We can see on Figure 6 that the parameter $1 / F$ obtained from relation (14) (solid black line) is correlated to the estimation of the speck's size made using the 2D-autocorrelation operation, despite some deviation for smaller particles. It is well known that there is a correction factor between the width of a mathematical signal and the width of its autocorrelation, which is classicaly done in ultra-short pulse measurement for example. This factor depends on the mathematical signal under consideration and can not be determined "a priori" in the case of our speckle-like pattern. We determine it "a posteriori". We have plotted on Figure 6 the parameter $\alpha / F$ versus $\Delta$ in dotted line with a correction factor $\alpha=0.9$. Assuming thus a correction factor of 0.9 , we can reduce the deviation for smaller particles, and we can see that it is possible to estimate the length of the stick versus the speck's size of the interferometric out-of-focus image. Let us now discuss the principal source of noise which brings uncertainty in the determination of the particle's size.

The main source of noise is the possibility to obtain 14 glare points located in a stick of dimension Length $_{1} \mu \mathrm{m} \times 10 \mu \mathrm{m} \times 10 \mu \mathrm{m}$, while the $15^{\text {th }}$ glare point is isolated, leading to a global stick of dimension Length $_{2} \mu \mathrm{m} \times 10 \mu \mathrm{m} \times 10 \mu \mathrm{m}$ with Length 2 significantly higher than Length $h_{1}$. In this case, the intensity due to the $15^{\text {th }}$ isolated glare point is not sufficient to influence significantly the width of the 2D-autocorrelation peak. The estimation of this width will then lead to an erroneous dimension of the stick: length $L e n g t h_{1}$ and not $L e n g t h_{2}$. Note that such cases are regularly present on Figure 6. This effect is reduced when the number of glare points is increased, because the possibility to observe an isolated glare point is then reduced. However,

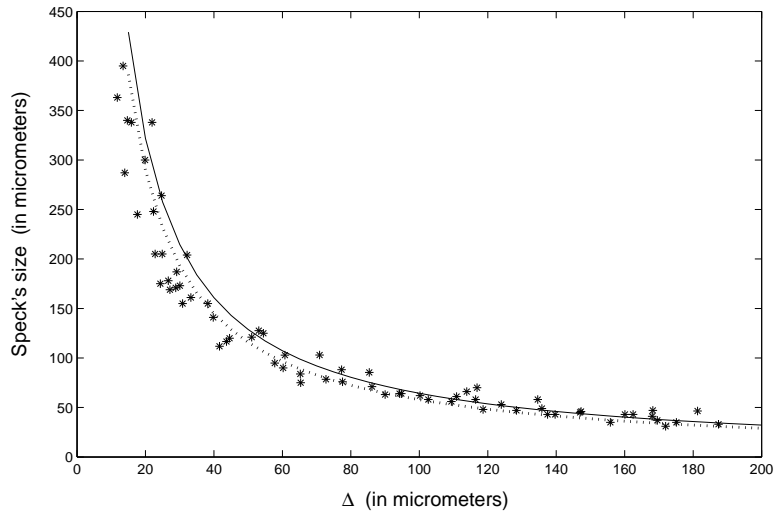

FIG. 6 Evaluation of the speck's size of the speckle pattern versus the distance between the two farthest emitters describing the scattering object $\Delta$ : (a) Parameter $1 / F$ obtained from relation (14) (solid black line); (b) Parameter $\alpha / F$ versus $\Delta$ (dotted line) with a correction factor $\alpha=0.9$; (c) Width of the central peak of the 2D-autocorrelation of the corresponding speckle pattern (crosses).

it does not modify the general law given by relation (14). Although our description of the scattering objects is based on important assumptions, we can see that the evaluation of parameters as the speck size of the speckle-like patterns gives the possibility of evaluating quantitatively the dimension of scattering irregular rough particles. As our simulator is based on optical ABCD transfer matrices, we can describe a wide range of imaging systems (with different kinds of objectives, cylindrical lenses, for in situ measurements through transparent pipes...). Let us now discuss the domain of investigation.

\subsection{Domain of investigation}

As with classical ILIDS for droplets, a given set-up has a limited domain of particle size determination. When the defocus parameter is sufficiently high, the higher-size limit is due to the pixel size on the CCD sensor and to Nyquist sampling theorem. We must indeed satisfy $F<1 /\left(2 * \delta_{C C D}\right)$ where $\delta_{C C D}$ is the CCD pixel size, and F is given by relation (14), with $\Delta$ the largest dimension of the particle (i.e. the distance between the two farthest glare points). When the defocus parameter is too small, the higher-size limit is not limited by sampling theorem, but by the aperture of the system. The same problem occurs in classical ILIDS with droplets when the defocus is such that the out-of-focus images of the 2 glare points do not overlap anymore. Typically, for the configuration under study (parameters $z_{1}=0.13 \mathrm{~m}, f=0.1 \mathrm{~m}, z_{2}=0.393 \mathrm{~m}, R_{0}=10 \mathrm{~mm}$ ), Nyquist theorem limits the higher particle size to $320 \mu \mathrm{m}$ for a CCD pixel size of $10 \mu \mathrm{m}$. In this case, the out-of-focus images of all glare points of the irregular particle overlap and the previous analysis is correct.

The lower-size limit is due to the aperture of the out-offocus image of the particle. It is well known that it is not possible to measure the diameter of a droplet with ILIDS when there is only one fringe within the out-of-focus image. The same limit occurs here. It is not possible to measure the dimension of small particles whose out-of-focus images only exhibit one brilliant or dark fringe. To illus- 
(a)
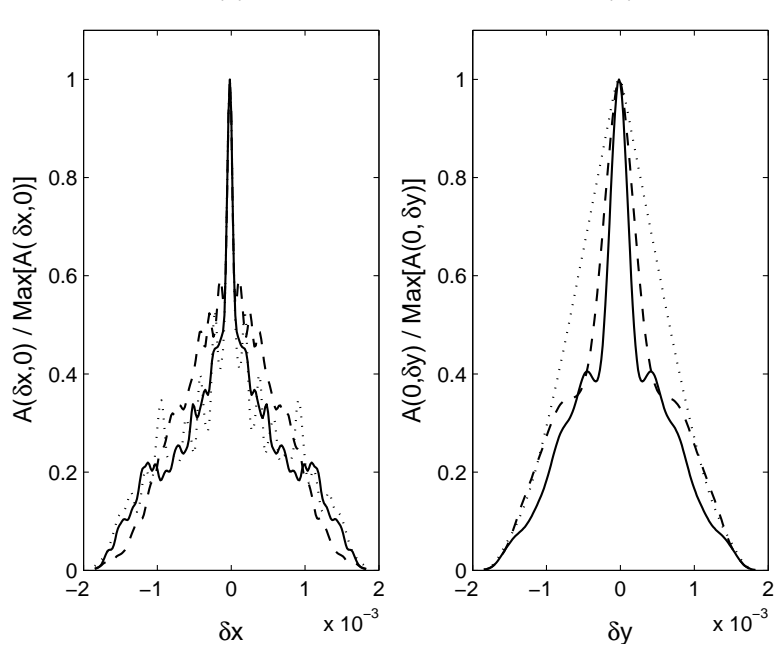

FIG. 7 x-cut (a) and $y$-cut (b) of the central peak of the 2D-autocorrelation images considering 15 emitters along a stick whose width is $20 \mu \mathrm{m}$ (solid lines), $10 \mu \mathrm{m}$ (dashed lines) or $2 \mu \mathrm{m}$ (dotted lines).

trate this, Figure 7 shows the $x$-cut and $y$-cut of the 2Dautocorrelation peak obtained from 3 different interferometric out-of-focus images of sticks. These three sticks are represented by 15 glare points randomly located in a parallelepipede of dimensions $(80 \mu \mathrm{m} \times 20 \mu \mathrm{m} \times 20 \mu \mathrm{m})$ for the stick $1,(80 \mu \mathrm{m} \times 10 \mu \mathrm{m} \times 10 \mu \mathrm{m})$ for the stick 2 , and $(80 \mu \mathrm{m} \times 2 \mu \mathrm{m} \times 2 \mu \mathrm{m})$ for the stick 3 . The results are reported in solid lines for the stick 1 , in dashed lines for the stick 2 , in dotted lines for the stick 3 . For the three sticks, there is no difference on the $\mathrm{x}$-cut of the central 2D-autocorrelation peak. This is not surprising as the stick exhibits in all cases a $x-$ dimension equal to approximately $80 \mu \mathrm{m}$. The $\mathrm{y}$-axis cuts are completely different. According to relation (14), the lower the $y$-dimension of the stick, the higher the width of the y-cut autocorrelation central peak. We can however note that the y-cut for the stick 3 whose y-dimension is only $2 \mu \mathrm{m}$ (dotted line) is like a triangular function, which corresponds to the autocorrelation of a squared-signal. In this case, there is only a brillant fringe along the y-axis of the whole out-of-focus image, and the $y$-cut of the 2D-autocorrelation peak does just show the $y$ axis autocorrelation of the envelope of the out-of-focus image. For this configuration, the lower-size limit is a particle size of $8 \mu \mathrm{m}$. For particles smaller than $8 \mu \mathrm{m}$, we observe only one brillant or dark fringe, and the y-cut of the 2D-autocorrelaton is a triangular function which represents the autocorrelation of a squared-signal (i.e. the out-of-focus image of the aperture). An example is observed in dotted line on Figure 7(b).

\section{EXPERIMENT}

In order to confirm these predictions, we have realized interferometric out-of-focus experiments. The set-up we realized is described in Figure 8. Particles are illuminated by a collimated laser beam. The laser is a frequency-doubled Nd:YAG laser emitting $10 \mathrm{~mJ}, 4 \mathrm{~ns}$ pulses at $532 \mathrm{~nm}$. The CCD sensor is a Marlin camera (pixel size $3.45 \mu \mathrm{m}$ ). Out-of-focus imaging of particles is realized with a spherical lens. The total transfer

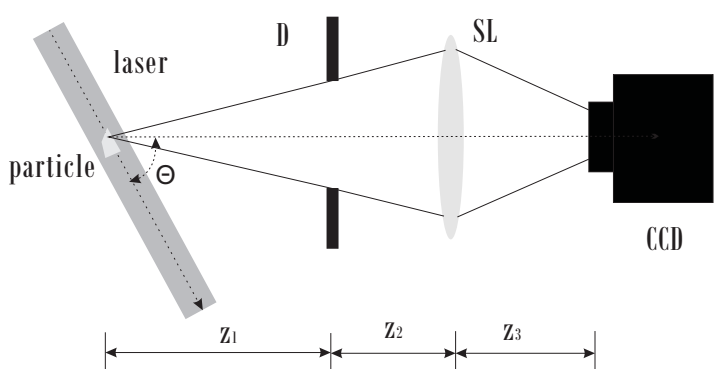

FIG. 8 Experimental interferometric out-of-focus imaging configuration.

matrix of the imaging system is given by

$$
M_{\text {tot }}^{p}=M_{z_{3}} \times M_{S L} \times M_{z_{1}+z_{2}}
$$

Matrix $M_{z_{1}+z_{2}}$ (resp. $M_{z_{3}}$ ) corresponds to propagation in air through distance $z_{1}+z_{2}$ (resp. $z_{3}$ ). $z_{1}$ is the distance between the scattering objects and the plane of the aperture. $z_{2}$ is the distance fom the aperture to the spherical lens (SL). $z_{3}$ is the distance between the spherical lens and the CCD sensor. Matrix $M_{S L}$ is the transfer matrix of the spherical lens. The different parameters of this system are: $z_{1}=85 \mathrm{~mm}, z_{2}=63 \mathrm{~mm}$, $z_{3}=195 \mathrm{~mm}, f_{S L}=100 \mathrm{~mm}$. The diameter of the spherical lens is $9.6 \mathrm{~mm}$. The particles that we consider are $\mathrm{NaCl}$ salt crystals. The image of a salt crystal is presented on Figure 9. Each graduation represents $100 \mu \mathrm{m}$. The image has been captured with a Nikon numerical camera. For this picture, the crystal is illuminated in reflection by an annular incoherent white lamp, located around the objective of the camera. The particle appears as an irregular ovoid particle. Figure 10(a) shows an experimental out-of-focus pattern that we obtain. It is a speckle-like pattern. We realize a second-order 2D-autocorrelation of this pattern. It is presented in Figure 11(a) (Figure 11(b) is a zoom of the central peak). The central peak is not circular but ellipsoidal indicating an asymetry of the salt crystal (in the $(x, y)$ plane, at the time when the image has been captured). The symmetry axes of the central peak are the $x$ - and y-axes of the CCD sensor (uncertainty of $\pm 2^{\circ}$ ). Figure 12 shows the $x$-cut (Figure 12(a)) and y-cut (Figure 12(b)) of the central peak of this 2D-autocorrelation (in dotted lines). Based on these traces and on the parameters of the experimental set-up (which give $\left.B_{\text {tot }}^{x, y}\right)$, we can estimate the salt crystal dimensions along both $\mathrm{x}-$ and y-axes using relation (14). We obtain $600 \mu \mathrm{m}$ along $\mathrm{x}$, and $400 \mu \mathrm{m}$ along $\mathrm{y}$. We have then simulated the interferometric out-of-focus image that we should obtain considering an ellipsoidal particle: it is represented by the random location of 80 glare points on an ellipsoid of dimensions $600 \mu \mathrm{m}$ along $\mathrm{x}$ and $400 \mu \mathrm{m}$ along $\mathrm{y}$ and $\mathrm{z}$. Note that the dimension along $\mathrm{z}$ has no significant influence as the image captured by the CCD sensor is parallel to the $(x, y)$ plane. We could verify that we would obtain the same results if this z-dimension had another value in the range $[0 \mu \mathrm{m}, 600 \mu \mathrm{m}]$. We present on Figure 10(b) the pattern predicted using relations (5)-(10). In order to simulate experimental conditions, we have added a continuous background signal equivalent to 10 percent of the maximal intensity of the pattern. We have then calculated the second-order 2D-autocorrelation of the pattern. We present on Figure 12 the x-cut (Figure 12(a)) and y-cut (Figure 12(b)) of the central peak of the 2D-autocorrelation (in solid lines). They can be compared with the experimental curves already reported in dotted lines. Although the speckle patterns are not identi- 


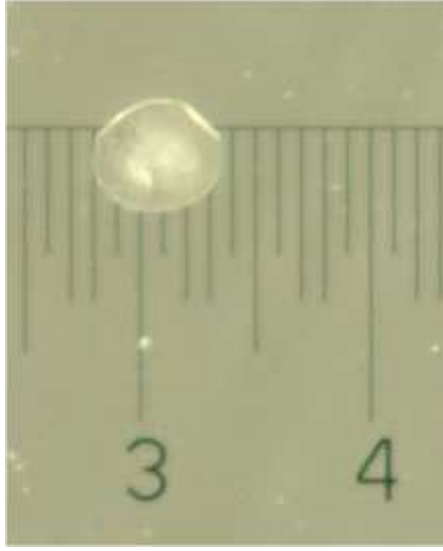

FIG. 9 Image of a typical $\mathrm{NaCl}$ salt crystal.
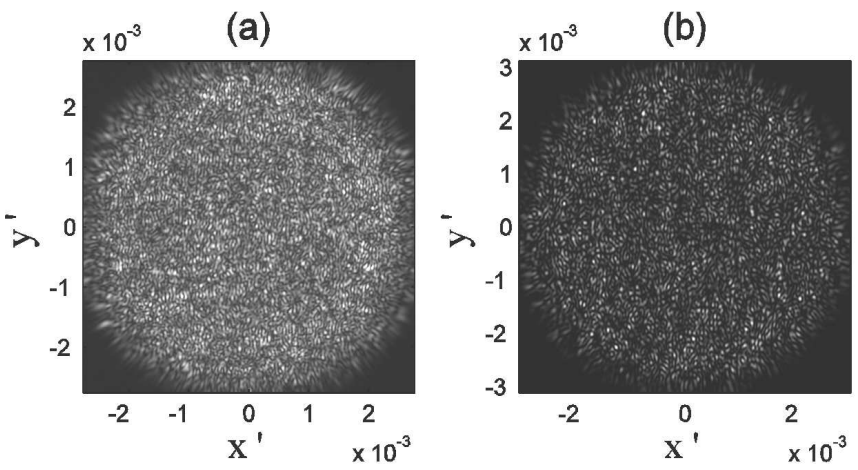

FIC. 10 Experimental interferometric out-of-focus pattern recorded with a $\mathrm{NaCl}$ salt crystal (a), and simulated out-of-focus pattern (b). (dimensions in meters).

cal, the characteristics of the central peak of the second-order 2D-autocorrelation are in good agreement $(x-$ and $y$-sizes of the speck). In conclusion, it is possible to interpret specklelike out-of-focus patterns and to give some characteristics of the particle under study, although we are not able to describe exactly the scattering properties of the particle.

\section{CONCLUSION}

We have thus developed a simulator of interferometric out-offocus images for the analysis of irregular rough particles. The rigorous description of the scattering properties of irregular objects using exact Lorenz-Mie theory does not have exact solutions in a wide number of cases. We think that, although we are not able to describe rigorously the scattering properties, we are able to predict some statistical properties of the scattered images using a simplified approach: i.e. the dimension of the central peak of the 2D-autocorrelation of the specklelike patterns. Our method consists of describing complex irregular objects by a random location of glare points along the global form of the irregular objects. We have shown that it is possible to predict some simple morphological properties of the particle (morphology of the repartition of glare points for more precision). Simulations have been confirmed by experimental images recorded with $\mathrm{NaCl}$ salt crystals. Although our description of the scattering properties is not exact, the images that we predict contain exact informations about the particle (a)

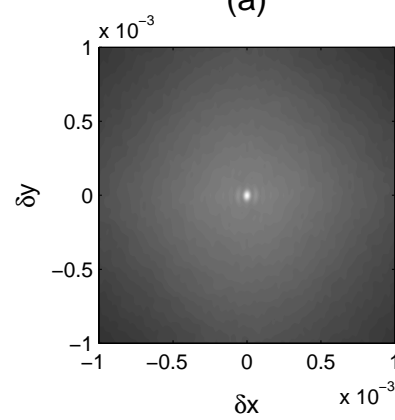

(b)

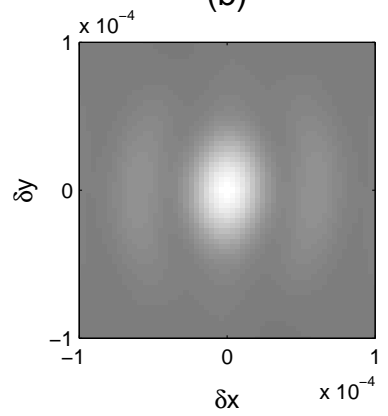

FIC. 11 2D-autocorrelation of the experimental out-of-focus pattern of Figure 10(a), and zoom of the central peak of this autocorrelation (b). (dimensions in meters). (a)

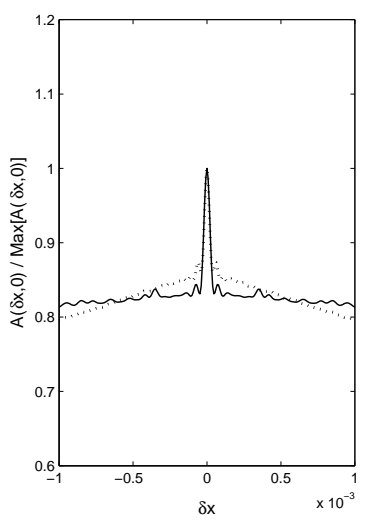

(b)

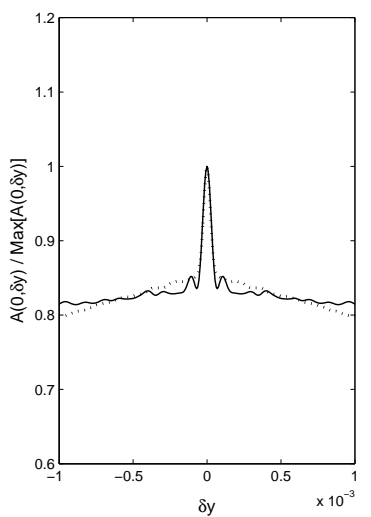

FIG. $12 x$-cut (a) and y-cut (b) of the central peak of the 2D-autocorrelation calculated from the experimental out-of-focus pattern (dotted lines) and the simulated out-offocus pattern (solid lines).

under study (through the analysis of the 2D-autocorrelation of the speckle-like patterns).

In our simulator, the imaging system is described by optical transfer matrices. We can thus describe any system (systems involving cylindrical lenses, in situ measurements in pipes, presence of windows to protect the CCD sensor...). We can clearly differentiate the influence of the imaging system and of the particle under study. We obtain analytical relations which link the dimension of the particle to the size of the light speck (through a coefficient of the global transfer matrix, and thus for a wide range of imaging systems).

Despite the very important simplification that we make to describe the scattering processes, we think that our simulator is a very convenient tool for the analysis of irregular particles in interferometric out-of-focus imaging experiments. It allows to give some quantitative informations (size, global shape) in domains where there are no theoretical models to describe the scattering properties of such irregular particles. It should allow to develop and calibrate real-time algorithms to detect the dimension of irregular particles, and to give some simple morphological informations about the particle. Using double- 
acquisition schemes, it should allow to measure and interpret the size and velocity of particles, combined to the analysis of their rotation in a flow. At this stage, in order to prove the concept, we have limited our analysis to simple morphological aspects of the particles (width and height). But we think that this simulator should allow to obtain more details concerning the form of the particle in the future (as the existence of specific symmetries of the object for example). Another important perspective is the extension to multifrequency interferometric particle imaging to enhance the accuracy of the characterization of the particles [25].

\section{ACKNOWLEDGEMENTS}

The authors acknowledge the financial support of the french Agence Nationale de la Recherche (ANR), through the program Investissements d Avenir (ANR-10-LABX-09-01) , LabEx EMC3, and the European commission through the EUFAR project.

\section{References}

[1] G. König, K. Anders, and A. Frohn, "A new light-scattering technique to measure the diameter of periodically generated moving droplets," J. Aerosol Sci. 17, 157-167 (1986).

[2] R. Ragucci, A. Cavaliere, and P. Massoli, “Drop Sizing by laser light scattering exploiting intensity angular oscillation in the Mie regime," Part. Part. Syst. Char. 7, 221-225 (1990).

[3] K. H. Hesselbacher, K. Anders, and A. Frohn, “Experimental investigation of Gaussian beam effects on the accuracy of a droplet sizing method," Appl. Optics 30, 4930-4935 (1991).

[4] A. R. Glover, S. M. Skippon, and R. D. Boyle, "Interferometric laser imaging for droplet sizing: a method for droplet-size measurement in sparse spray systems," Appl. Optics 34, 8409-8421 (1995).

[5] N. Damaschke, H. Nobach, and C. Tropea, "Optical limits of particle concentration for multi-dimensional particle sizing techniques in fluid mechanics," Exp. Fluids 32, 143-152 (2002).

[6] T. Kawaguchi, Y. Akasaka, and M. Maeda, "Size measurements of droplets and bubbles by advanced interferometric laser imaging technique," Meas. Sci. Technol. 13, 308-316 (2002).

[7] J. V. Dave, "Scattering of visible light by large water spheres," Appl. Optics 8, 155-164 (1969).

[8] H. C. Van de Hulst, Light Scattering by Small Particles (Dover Publications, Mineola, 1981).

[9] G. Lacagnina, S. Grizzi, M. Falchi, F. Di Felice, and G. P. Romano, "Simultaneous size and velocity measurements of cavitating microbubbles using interferometric laser imaging," Exp. Fluids 50, 1153-1167 (2011).
[10] Y. Hardalupas, S. Sahu, A. M. K. P. Taylor, and K. Zarogoulidis, "Simultaneous planar measurement of droplet velocity and size with gas phase velocities in a spray by combined ILIDS and PIV techniques," Exp. Fluids 49, 417-434 (2010).

[11] W. J. Glantschnig, and S. H. Chen, "Light scattering from water droplets in the geometrical optics approximation," Appl. Optics 20, 2499-2509 (1981).

[12] H. Shen, S. Coetmellec, G. Grehan, and M. Brunel, "ILIDS revisited: elaboration of transfer matrix models for the description of complete systems," Appl. Optics 51, 5357-5368 (2012).

[13] M. Maeda, Y. Akasaka, and T. Kawaguchi, "Improvements of the interferometric technique for simultaneous measurement of droplet size and velocity vector field and its application to a transient spray," Exp. Fluids 33, 125-134 (2002).

[14] A. Querel, P. Lemaitre, M. Brunel, E. Porcheron, and G. Gréhan, "Real-time global interferometric laser imaging for the droplet sizing (ILIDS) algorithm for airborne research," Meas. Sci. Technol. 21, 015306 (2010).

[15] D. Lin, N. C. Angarita-Jaimes, S. Chen, A. H. Greenaway, C. E. Towers, and D. P. Towers, "Three dimensional particle imaging by defocusing method with an annular aperture," Opt. Lett. 33, 905-907 (2008).

[16] H. Shen, S. Coetmellec, and M. Brunel, "Cylindrical interferometric out-of-focus imaging for the analysis of droplets in a volume," Opt. Lett. 37, 3945-3947 (2012).

[17] H. Shen, S. Coetmellec, and M. Brunel, "Simultaneous 3D location and size measurement of spherical bubbles using cylindrical interferometric out-of-focus imaging," J. Quant. Spectrosc. Radiat. Transf. 131, 153-159 (2013).

[18] C. Mounaim-Rousselle, and 0. Pajot, "Droplet sizing by interferometric Mie scattering in engine environment," Proc. SPIE 3172, 700-707 (1997).

[19] H. C. van de Hulst, and R. T. Wang, "Clare points," Appl. Optics 30, 4755-4763 (1991).

[20] C. Palma, and V. Bagini, "Extension of the Fresnel transform to ABCD systems," J. Opt. Soc. Am. A 14, 1774-1779 (1997).

[21] A. J. Lambert, and D. Fraser, "Linear systems approach to simulation of optical diffraction," Appl. Optics 37, 7933-7939 (1998).

[22] H. T. Yura, and S. G. Hanson, "Optical beam wave propagation through complex optical systems," J. Opt. Soc. Am. A 4, 1931-1948 (1987).

[23] J. J. Wen, and M. Breazeale, "A diffraction beam expressed as the superposition of Gaussian beams," J. Acoust. Soc. Am. 83, 1752-1756 (1988).

[24] J. W. Goodman, Speckle phenomena in optics. Theory and Applications (Roberts and Company Publishers, Greenwood Village, 2009).

[25] S. Dehaeck, and J. van Beeck, "Multifrequency interferometric particle imaging for gas bubble sizing," Exp. Fluids 45, 823-831 (2008). 